Ann. Biol. anim. Bioch. Biophys., I962, 2 (2), I57-I62

\title{
ACTION DES HORMONES GONADOTROPES SUR LE TESTICULE DE L'AGNEAU IMPUBÈRE. RÉPONSE PARTICULIÈRE DE LA LIGNÉE SERTOLIENNE
}

\author{
(NOTE PRÉLIMINAIRE) \\ M. COUROT \\ Avec la collaboration technique de Christiane Richetin \\ Station de Recherches de Physiologie animale, \\ Centre national de Recherches zootechniques, Jouy-en-Josas (Seine-et-Oise).
}

Le déterminisme du déclenchement et du maintien de l'activité spermatogénétique est encore mal connu. En particulier on n'a que des indications très vagues sur la nature des stimuli qui provoquent la multiplication des cellules germinales. Il est admis, en général, que les hormones gonadotropes ont une activité gamétostimulante (SMith, et LEONARD, I934; GreEP, FEvol, et Hisaw, I936 ; Simpson et ses collaborateurs, I946 à I96I ; Van OORdT, SLUITER et Van OORDT, I95 I ; MESS, I952 ; CLERMONT et MORGENTALER, I955 ; COURRIER, I956 ; RANDOLPH et al., I959); cependant le rôle précis de chacune d'entre elles dans les processus qui constituent l'ensemble complexe du cycle spermatogénétique n'est pas encore défini.

C'est pourquoi nous avons commencé l'étude de ce problème chez l'Agneau, des travaux antérieurs nous ayant permis de déterminer la chronologie de l'établissement de la spermatogenèse chez cet animal (CouroT, I962).

\section{MATÉRIEL E'T MÉTHODES}

Les expériences ont porté sur 37 Agneaux de race "lle-de-France " de I 5 à 2 I kg âgés de 50 à 75 jours. Ceux-ci ont reçu différents produits gonatropes (tableau I) en injection sous-cutanée (sauf l'hormone chorionique qui a été injectée par voie intrapéritonéale), matin et soir pendant 5 jours. L.es testicules ont été prélevées par castration 12 à 18 heures après la dernière injection, pesés puis puis fixés dans le mélange de Bouin-Hollande pour l'étude histologique selon les procédés déjà décrits (COUROT, 1962). 
TABLEAU I

Caractéristiques et doses des produits gonadotropes employés

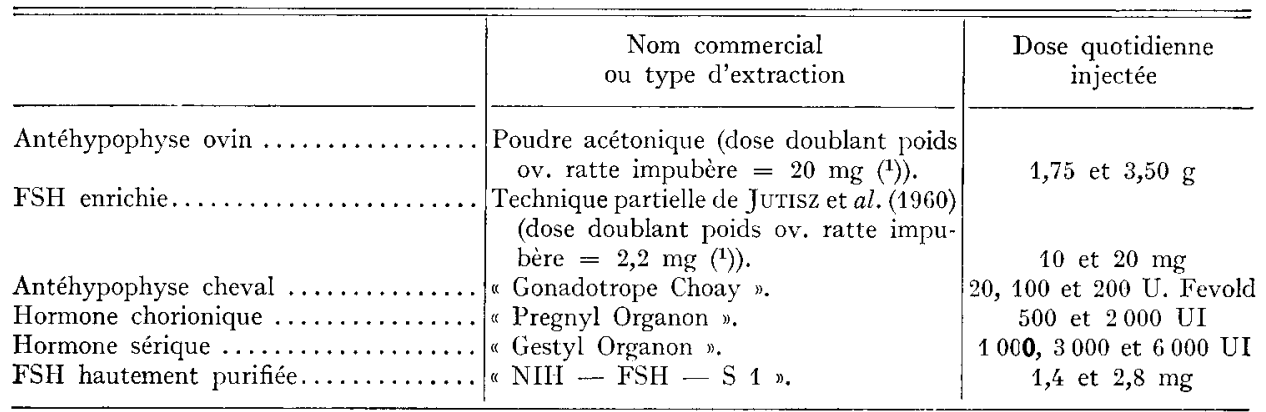

(1) Dosage suivant la technique de Steelman et Poilley (1953).

Dans la première expérience (tableau 2) nous avons injecté une suspension de poudre acétonique antéhypophysaire brute à 5 Agneaux et une préparation antéhypophysaire enrichie en principe folliculostimulant à 6 Agneaux.

Dans la seconde expérience (tableau 3) qui a porté sur 26 Agneaux, 5 d'entre eux ont reçu des injections d'hormone chorionique, 8 de l'extrait hypophysaire de cheval, 9 de l'hormone sérique et 4 de la FSH hautement purifiée, 3 Agneaux témoins ont reçu des injections de sérum physiologique.

Certaines doses injectées peuvent paraître très fortes mais il faut se rappeler que les animaux, bien qu'impubères, pèsent déjà 15 à $20 \mathrm{~kg}$.

\section{RÉSULTATS}

Les résultats obtenus sont présentés dans les tableaux 2 et 3 .

Les réponses pondérales des testicules sont assez variables. Dans 1'ensemble cependant on constate une augmentation du poids des testicules, la plus faible étant donnée par 1'hormone chorionique.

Par ailleurs le diamètre des cordons sexuels a subi un accroissement significatif dans la plupart des cas, les réactions les plus faibles ayant été obtenues avec l'hormone chorionique.

D'autre part, en aucun cas, les cellules germinales primordiales n'ont donné de spermatogonies en évolution et leur réponse est assez difficile à interpréter : en effet, les résultats présentés dans les tableaux 2 et 3 se rapportent à une coupe transversale de cordon sexuel de Iou d'épaisseur, c'est-à-dire à un volume pratiquement constant. Or le volume total des cordons sexuels par testicule a augmenté dans la plupart des cas. Nous avons donc calculé le nombre total des cellules germinales présentes dans le testicule entier : il a augmenté sous 1'effet de l'extrait hypophysaire total et de la préparation enrichie en FSH dans l'expérience I, mais il ne semble pas avoir été modifié par l'injection des autres prođuits gonadotropes utilisés dans l'expérience 2 .

Par contre la réaction la plus spectaculaire et la plus inattendue a été celle des cellules de la lignée sertolienne (Cellules de soutien): nous remarquons en effet une augmentation du nombre de ces cellules avec toutes les préparations gonadotropes injectées ; 1a 


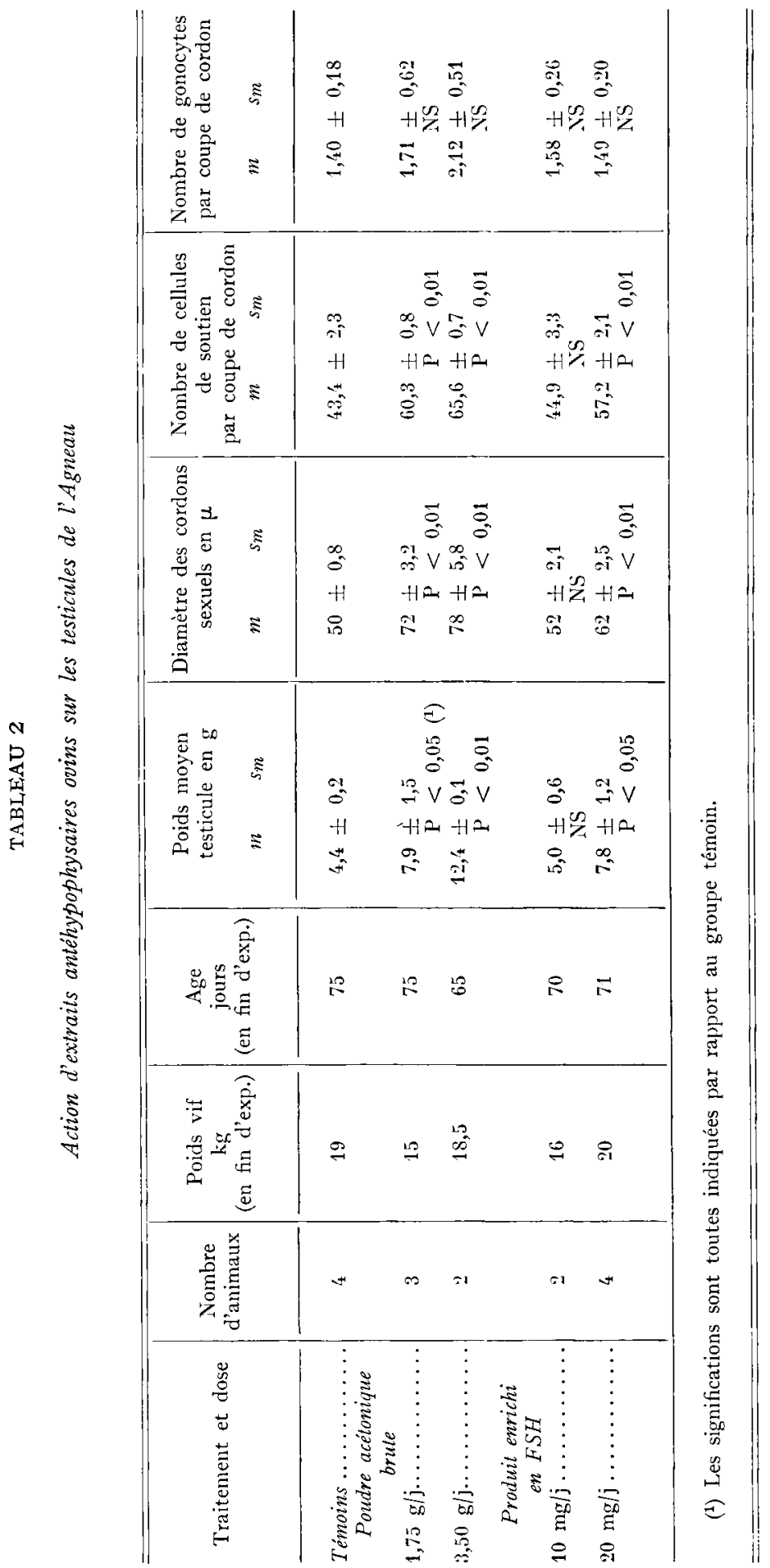




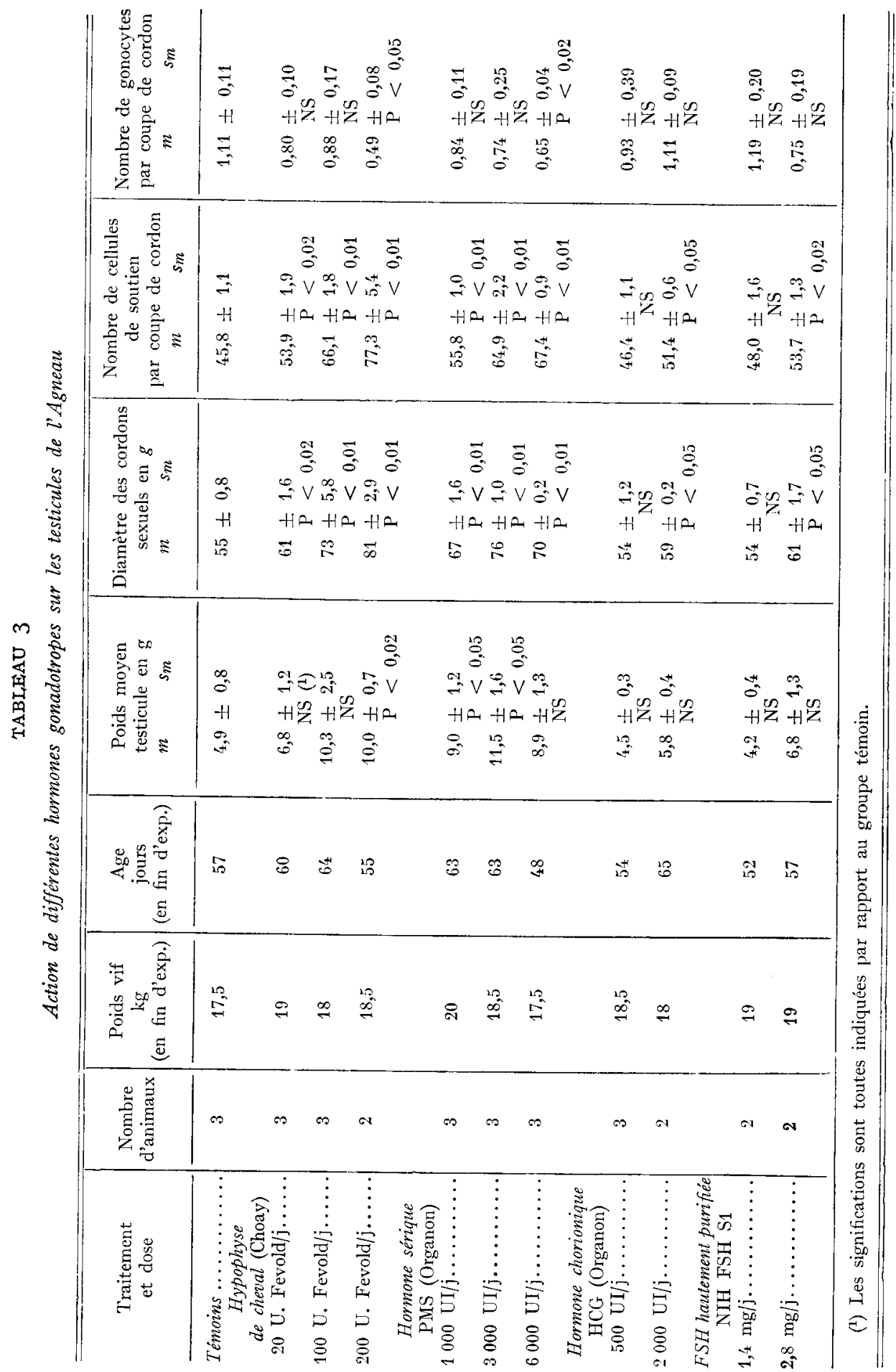


plus faible réponse est de nouveau obtenue avec 1'hormone chorionique et la plus forte fait suite à l'injection d'extrait antéhypophysaire de cheval. L'activité nucléaire des cellules de soutien est tout à fait exacerbée, les noyaux présentent en effet des formes extrêmement irrégulières, ils vont jusqu'à prendre l'allure de véritables figures d'amitose (fig. I).

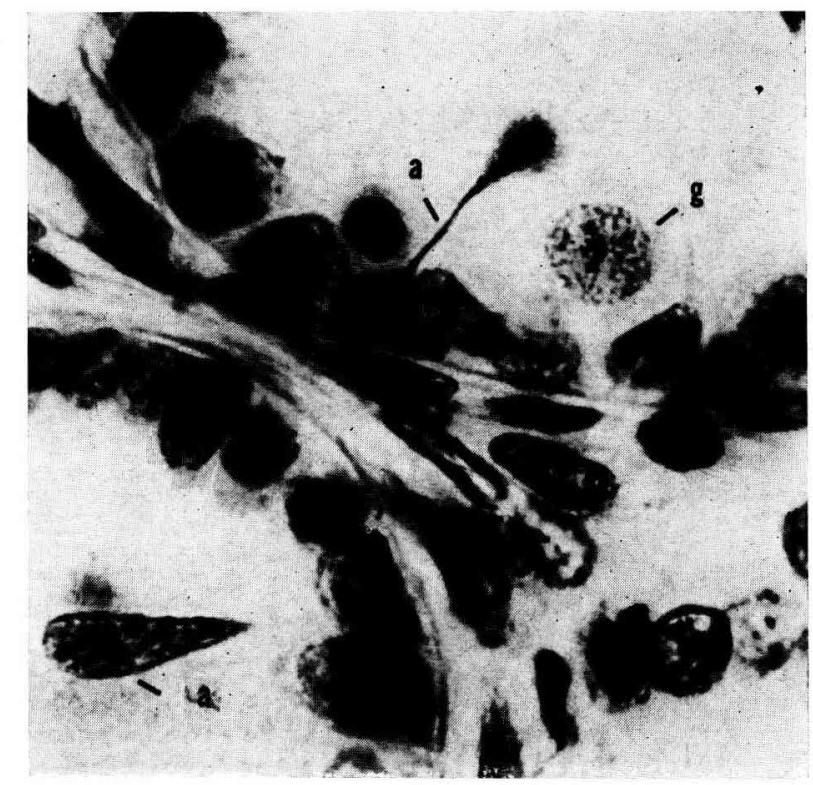

FIG I. - Cordon sexuel d'un testicule d'agneau ayant reçu des injections d'un extrait antéhy pophysaire ovin enrichi en FSH. Noter la stimulation des cellules de soutien dont certaines présentent des figures d'amitose $(a)$ alors que le gonocyte n'est pas modifié $(g)$ ( $x$ I $\infty \circ)$

\section{CONCLUSION}

Chez l'agneau dont les testicules ne manifestent encore aucune activité spermatogénétique, les hormones gonatrodopes employées ont agit sur les cordons sexuels dans les conditions expérimentales où nous avons travaillé. Certes ces hormones ont été incapables de provoquer le démarrage de la spermatogenèse et, seules, deux préparations d'extrait antéhypophysaire ovin ont entraîné une augmentation globale du nombre des gonocytes, mais elles ont toutes manifesté une action très nette sur les cellules de la lignée Sertolienne dont le nombre a augmenté et dont 1'activité nucléaire a été modifiée. Une réaction đe ce type avait déjà été mentionnée chez certains Batraciens (DEISOL, I960) mais n'avait pas encore été rapportée chez les Mammifères, du moins à notre connaissance. Cette action primaire des hormones gonadotropes paraît liée à l'activité "folliculo stimulante " des préparations puisque le produit hautement purifié, FSH-NIH-S I, a provoqué une telle réaction ; toutefois il ne nous est pas encore possible de conclure définitivement sur ce point. 


\title{
SUMMARY
}

\author{
ACTION OF GONADOTROPINS IN THE PREPUBERAL LAMB
}

37 "Ile-de-France " lambs, whose testes were not yet showing any spermatogenic activity, were given twice a day for five consecutive days, injections of different gonadotropic hormone preparations (hypophyseal extracts, PMS, HCG, FSH-NIH...).

In the conditions of the experiment no important evolution of the gonocytes was observed. However an important and fairly constant stimulation of the precursors ot the Sertoli cells was seen, probably dependant on the FSH activity of the preparations.

\section{REMERCIEMENTS}

Nous tenons à remercier très vivement le service « Endocrinology study section of the National Institutes of Health" qui nous a aimablement fourni l'hormone hautement purifiée FSH-NIH-S-I utilisée dans ce travail.

\section{RÉFÉRENCES BIBLIOGRAPHIQUES}

Clermont Y., Morgentaler H., 1955. Quantitative study of spermatogenesis in the hypophysectomized rat. Endocrinology 57, 369-382.

Courot M., rg62. Développement du testicule chez l'Agneau. Établissement de la spermatogenèse. Ann. Biol. anim. Bioch. Biophys., 2, 21-37.

Courrier R., r956. Remarque sur la greffe d'hypophyse. Arch. Biol. 6\%, 46r-469.

Dessol M., rg60. Sur l'action de l'hormone gonadotrope FSH sur le testicule du têtard d'Alytes obstetricans Laur. Durée de la spermatogenèse expérimentale. C. R. Soc. Biol., 154, 927-930.

Greep R. O., Fevold H. L., Hisaw F. L., 1936. Effects of two hypophyseal gonadotrophic hormones on the reproductive system of the male rat. Anat. Rec., 65, 261-27 I.

Jutisz M., Hermier C., Colonge A., Courrier R., I960. Isolement des hormones hypophysaires : purification de l'hormone folliculo-stimulante de Mouton. C. R. Acad. Sci., 250, 431-435.

MEss B., 1952. Influence of hypothalamic injury on spermatogenesis. Acta morphologica, Ac. Sci. Hung., 2, 275.285.

Van Oordt G. J., Sluiter J. W., Van Oordt P. G. W. J., I95I. Spermatogenesis in normal hypophysectomized frogs (Rana Temporia) following gonadotrophin administration. Acta Endocr., 7, 257-26.

SIMPSON M. E., 1959. Role of the anterior pituitary gonadotropins in reproductive processes. in "Reproduction in Domestic Animals n, Ed. Cole H. H., Cupps P. T., Vol 1, 6o-ro6 Acad. Press, New York and London.

Simpson M. E., Evans H. M., ig46. Comparison of the spermatogenic and androgenic properties of testosterone propionate with those of pituitary ICSH in hypophysectomized 40-day-old male rats. Endocrinology, 39, $28 \mathrm{I}-285$.

Simpson M. E., Li C. H., Evans H. M., 1951. Synergism between pituitary follicle stimulating hormone (FSH) and human chorionic gonadotrophin (HCG). Endocrinology., 48, 370-383.

Smith P. E., LeONARD S. L., 1934. Responses of the reproductive system of hypophysectomized and normal rats to injections of pregnancy urine extracts. I - the Male. Anat. Rec., 58, 145-I 73 .

Stenlman S. L., Pohley F. M., I953; Assay of the follicle stimulating hormone based on the augmentation with human chorionic gonadotropin. Endocrinology., 53, 604-6r6.

Woods M. C., Simpson M. E., 196r. Pituitary control of the testis of the hypophysectomized rat. Endocrinology., 69, 91-I25.

Randolph P. W., Lostroh A. J., Grattarola R., Squire P. G., Li C. H., i959. Effect of ovine interstitial cell-stimulating hormone on spermatogenesis in the hypophysectomized mouse. Endocrinology., 65, $433 \cdot 44$ I. 